\title{
Why we need to measure turbulent magnetic fields in HII regions
}

\author{
John E. Beckman ${ }^{1,2}$ and Mónica Relaño ${ }^{1,3,4}$ \\ ${ }^{1}$ Instituto de Astrofísica de Canarias, Calle Vía Láctea s/n, \\ E-38200 La Laguna, Tenerife, Spain \\ email:jeb@iac.es \\ ${ }^{2}$ Consejo Superior de Investigaciones Científicas, Madrid, Spain \\ ${ }^{3}$ Departamento de Física Teóretica y del Cosmos, Universidad de Granada, Spain \\ ${ }^{4}$ Institute of Astronomy, University of Cambridge, UK \\ email: mrelano@ast.cam.ac.uk
}

\begin{abstract}
We have measured the emission line profiles in $\mathrm{H} \alpha$ from populations of HII regions in nearby spiral galaxies, and extracted their non-thermal line widths. These are supersonic for the more luminous regions. We infer from plots of line width, $\sigma$, against $\mathrm{H} \alpha$ luminosity, a set of regions in virial equilibrium, and derive their masses summing all known components. The virial masses are considerably larger. If this discrepancy, and the supersonic line widths, are due to the presence of magnetic fields, we can estimate their strengths at a few microgauss. Observational confirmation is clearly required.
\end{abstract}

Keywords. HII regions - magnetic fields - turbulence

\section{Deriving the non-thermal line widths}

Using Fabry-Perot spectrometers (see Hernandez et al. 2008) we have measured emission line profiles in $\mathrm{H} \alpha$ from large populations of HII regions in a number of nearby galaxies (Relaño \& Beckman 2005; Relaño et al. 2005; Fathi et al. 2008). These have characteristic shapes: twin weak peaks in the wings (due to expanding shells, not the topic here), and a strong central peak. The widths of the latter include contributions from, (a) the instrumental width, (b) the natural line width, (c) the thermal width, and (d) the turbulent width. To derive (d) we subtract in quadrature components (a), (b), and (c) from the total width. For many HII regions these turbulent widths are considerably larger than the sound speed, (Rozas, Beckman \& Knapen 1996) of order $13 \mathrm{~km} \mathrm{~s}^{-1}$

\section{Turbulent line width v. Luminosity}

When we plot the turbulent line widths, $\sigma$, against the $\mathrm{H}$ luminosities, L, of the HII regions, (see Relaño \& Beckman 2005) we find: (a) A large scatter in for a given value of $\mathrm{L}$, but (b) a clear lower envelope in , which we assign to HII regions in virial equilibrium, while the others, the majority, have additional impulsive velocity components. Even for these virialized regions the virial masses are considerably higher than the masses estimated by summing over all known components (dark matter can be neglected on these scales). Finally we find that (c), the turbulent line widths on the virial envelope are in general supersonic.

It is important to note that we have gone far to include all mass components in our sum. Most relevant is that, in the more luminous HII regions the greater part of the gas is not ionized (Giammanco et al. 2004). Estimates of the HI contribution are of order $80 \%$ 
of the total gas mass, and over $70 \%$ of the total HII region mass (Chu \& Kennicutt 1994; Relaño et al. 2005). Even so the sum falls short of the virial mass by factors typically of 2 to 3 .

\section{The requirement for magnetic support}

An obvious way to account both for the apparently supersonic line widths and the excess mass derived using them is to postulate the presence of turbulent magnetic fields with the HII regions. It is at least conceptually plausible that turbulent plasmas give rise to turbulent fields. If we assume equipartition between kinetic and magnetic energies we obtain fields of between a few, and a few tens of microgauss. There is easily enough energy input from the ionizing stars to maintain fields of these strengths (see Myers \& Goodman 1988). It would clearly be of great interest to measure these, and some recent techniques are promising in that respect.

\section{References}

Beckman, J. E. \& Relaño, M. 2004, ApJS 292, 111

Chu, Y.-H. \& Kennicutt, R. C., Jr. 1994, ApJ 425, 720

Fathi, K., et al. 2008, ApJ 675, L17

Giammanco, C., Beckman, J. E., Zurita, A., \& Relaño, M. 2004, A\& $A$ 424, 877

Hernandez, O., et al. 2008, PASP 120, 665

Myers, P. C. \& Goodman, A. A. 1988, ApJ 329, 392

Relaño, M., Beckman, J. E., Zurita, A., Rozas, M., \& Giammanco, C. 2005, A\&A 431, 235

Relaño, M. \& Beckman, J. E. 2005, A\&A 430, 911

Rozas, M., Beckman, J. E., \& Knapen, J. H. 1996, A\& A 307, 735 